\title{
A case of atrial septal defect presenting with recurrent syncope
}

\author{
Seng Wee Cheo, Qin Jian Low \\ Cheo SW, Low QJ. A Case of Atrial Septal Defect Presenting with Recurrent Syncope. Malays Fam Physician. 2021;16(2);90-93. \\ https://doi.org/10.51866/cr1125
}

\section{Keywords:}

Atrial septal defect,

congenital heart disease,

pulmonary hypertension,

syncopal attack.

\section{Authors:}

\section{Cheo Seng Wee \\ (Corresponding author) \\ MRCP (UK)}

Department of Internal Medicine Hospital Lahad Datu, Lahad Datu Sabah, Malaysia

Email: cheosengwee@gmail.com

Low Qin Jian

MRCP (UK)

Department of Internal Medicine Hospital Sultanah Nora Ismail

Jalan Korma, Taman Soga,

Batu Pahat, Johor, Malaysia

\begin{abstract}
Atrial septal defect (ASD) is a congenital heart disease often encountered in the adult population, as it is frequently asymptomatic in childhood. In untreated patients with ASD, some may go on to develop complications such as atrial arrythmias, pulmonary hypertension and Eisenmenger syndrome. Pulmonary hypertension is seen in $6-35 \%$ of this group of untreated patients in adulthood as a result of left-to-right shunting. Symptoms of pulmonary hypertension include progressive dyspnoea, ascites and syncope. Here, we would like to illustrate a case of ASD presenting with recurrent syncopal attack.
\end{abstract}

\section{Introduction}

Atrial septal defect (ASD) is one of the most common congenital heart diseases in adults. It is characterised by the presence of communication between the two atria. ${ }^{1}$ Most ASDs are asymptomatic until the fourth decade of life. ${ }^{2}$ Some present with fatigue, dyspnoea on exertion, exercise intolerance or, occasionally, syncopal attack. ${ }^{3}$ Others may go on to develop complications such as atrial arrhythmias, paradoxical embolism and pulmonary hypertension. ${ }^{4}$ Here, we would like to illustrate a case of ASD presenting with recurrent syncope.

\section{Case Presentation}

A 14-year-old girl with no past illness presented to a local health clinic with recurrent syncopal attacks over a period of two months. These attacks were described as transient loss of consciousness on exertion. She had experienced four episodes of syncope over the past two months, each lasting a few minutes and associated with palpitation and decreased effort tolerance. Attacks were brief and the patient soon regained consciousness. Otherwise, she had no chest pain, orthopnoea, leg swelling or fever. There was no family history of recurrent syncope or sudden cardiac death, and the patient did not complain of any neurological symptoms. She did not seek treatment for the first three episodes of syncope, but after the fourth episode she went to a local health clinic to seek treatment.

Upon assessment, her blood pressure was
$102 / 73 \mathrm{mmHg}$, pulse rate was 81 beats per minute and saturation was $96 \%$ on room air. No cyanosis was noted. There was no postural drop in blood pressure and her pulses were regular. She exhibited no features of dysmorphism or clubbing of digits, and her jugular venous pressure was not raised. She had a non-displaced apex beat, but there was parasternal heave. Auscultation of the heart revealed a loud P2 with splitting of the second heart sound. There was no murmur heard. Lung and neurological examination were unremarkable.

The patient was then referred to hospital for further evaluation. Initial blood investigation revealed normal full blood count, renal profile, liver function test and electrolytes. Her electrocardiogram (ECG) showed sinus rhythm, right axis deviation, a tall tented $\mathrm{P}$ wave, deep $\mathrm{T}$ inversion from $\mathrm{V} 1$ to $\mathrm{V} 5$ and right ventricular hypertrophy (Figure 1). Congenital heart disease was then suspected based on the clinical signs and ECG findings. Our differential diagnosis was cardiac arrythmia, hypertrophic obstructive cardiomyopathy or orthostatic hypotension.

A transthoracic echocardiography was performed and showed that the patient had an atrial septal defect measuring $1 \mathrm{~cm}$, with right-to-left shunt from colour flow and a Qp:Qs ratio of 2.9. She had pulmonary hypertension, as evidenced by a dilated right atrium and right ventricle with estimated pulmonary pressure of $44 / 24 \mathrm{mmHg}$ (Figure 2). The patient was then referred to a cardiac 
centre, where a right heart catherization showed irreversible pulmonary hypertension not suitable for closure. She was started on a medical therapy of sildenafil and bosentan and, as of her last follow up, is doing well.

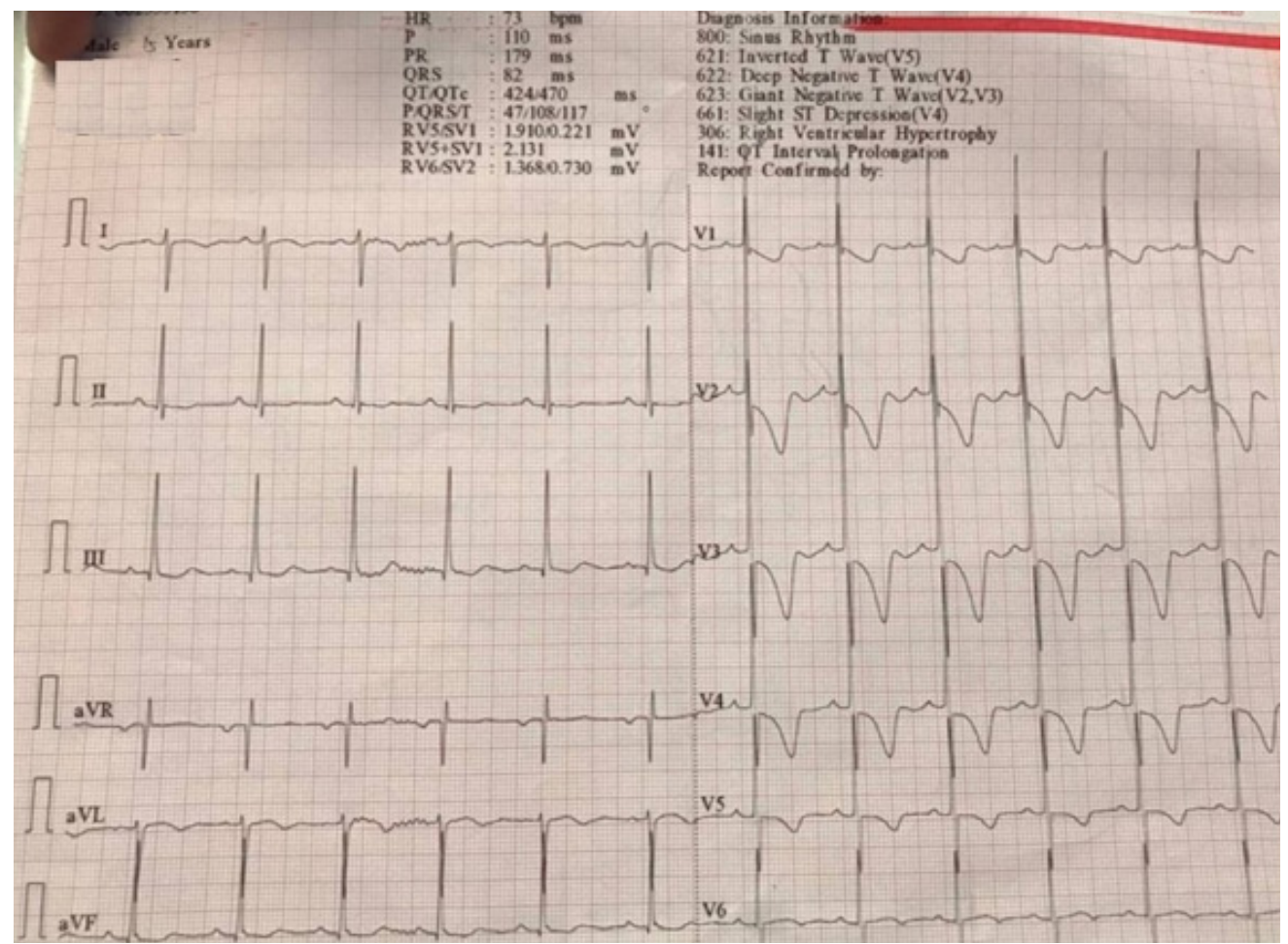

Figure 1: Electrocardiogram of 14-year-old female patient with syncope. Image shows sinus rhythm, right axis deviation, tall tented $\mathrm{P}$ wave, deep $\mathrm{T}$ inversion from $\mathrm{V} 1$ to $\mathrm{V} 5$ and right ventricular hypertrophy.

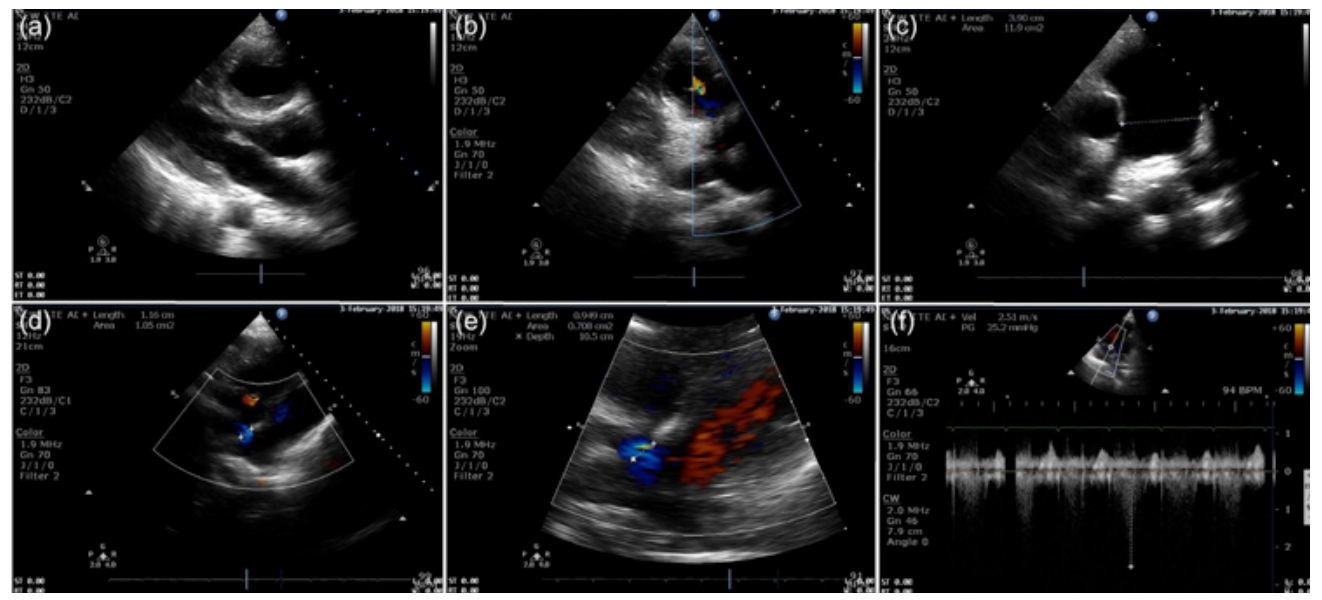

Figure 2: Transthoracic echocardiography images of 14-year-old female patient with syncope. (a) Parasternal long axis view showing dilated right ventricle. (b) Right ventricular outflow tract (RVOT) view showing pulmonary regurgitation. (c) Parasternal short axis at RVOT showing dilated pulmonary artery. (d) and (e) Subcostal view showing an atrial septal defect shunt. (f) Apical view showing tricuspid regurgitation.

\section{Discussion}

ASD is a relatively common congenital heart disease, with a birth prevalence of 1.6 per 1000 live births and a probability of survival into adulthood of $97 \% .^{5}$ It contributes to 30 $-40 \%$ of congenital heart disease (CHD) and is the third most common CHD. ${ }^{6}$ Broadly,
ASD can be divided into four types: ostium secundum $(60-70 \%)$, ostium primum $(15-$ $20 \%)$, sinus venosus $(5-15 \%)$ and coronary sinus $(<2 \%){ }^{7}$ Most ASDs are sporadic with no specific cause. Some ASDs, however, occur in association with genetic syndromes such as Noonan syndrome, Holt-Oram syndrome and 
Down's syndrome. ${ }^{6}$ Importantly, ASD remains an important diagnostic entity in the adolescent and adult patient, with considerable morbidity and mortality if left unrepaired.

Clinically, patients can have various presentations, and symptoms at presentations are often nonspecific. Patients with ASDs are usually asymptomatic in infancy and childhood. ${ }^{2}$ Infants may present with feeding difficulties, difficulty in breathing, growth retardation and recurrent chest infection, and some children may present with exertional dyspnoea and fatiguability. ${ }^{2,8}$ It is important to note that most patients remain asymptomatic until after their fourth decade. At that time, these adults may present with exertional dyspnoea, palpitations and fatiguability, symptoms that are likely due to heart failure, pulmonary hypertension, atrial tachyarrhythmias and co-existent mitral valve disease. ${ }^{7}$ Some patients may go on to develop pulmonary hypertension and Eisenmenger syndrome due to reversal of the shunt. ${ }^{\text {? }}$

Our patient presented with recurrent syncope on exertion. Each episode was associated with palpitation and she also complained of decreased effort tolerance. Her history was suggestive of a cardiac cause of syncope. Her ECG showed right axis deviation and right ventricular hypertrophy consistent with pulmonary hypertension. Subsequent echocardiography confirmed the diagnosis of pulmonary hypertension secondary to ASD. We believe that her recurrent syncope was likely due to pulmonary hypertension from her unrepaired ASD. Syncope in pulmonary hypertension is likely due to the loss of ventricular adaptation to afterload. ${ }^{10}$

Diagnosis of ASD is mainly achieved by echocardiography, either transthoracic or transoesophageal. ${ }^{5}$ Echocardiography can establish the size and location of the ASD, as well as the magnitude of shunting and presence of pulmonary hypertension. ${ }^{2}$ Electrocardiography findings of ASD include atrial arrythmias and right bundle branch block, among others. Long-term complications of uncorrected ASDs include paradoxical embolization, heart failure, pulmonary hypertension and Eisenmenger syndrome. 5 Secundum ASDs can be treated surgically or percutaneously, and closure is indicated when there is evidence of right ventricular compromise with or without symptoms. ${ }^{9}$ Our patient underwent right heart catheterization and was diagnosed with irreversible pulmonary hypertension. She was then treated with medical therapy.

\section{Conclusion}

Although ASDs are common, they remain very much underdiagnosed, as most are asymptomatic. This case highlighted the importance of early diagnosis of ASD, as early interventions can help in preventing the development of complications. In addition, this case also showed that structural heart disease is a potential cause when evaluating recurrent syncope.

\section{Acknowledgements}

The authors would like to thank the Malaysian Director of Health for permission to publish this paper.

\section{Conflicts of Interest}

The author declares no conflicts of interest.

\section{Funding}

The author received no financial support for this publication.

How does this paper make a difference to general practice?

- Recurrent syncope is a common clinical problem that we face in our daily clinical practice. This case highlighted the importance of clinical history and physical examination in evaluating recurrent syncope.

- Common causes of recurrent syncope include cardiac arrythmias, structural heart disease and ischemic heart disease. Electrocardiography is important in evaluating recurrent syncope.

- An abnormal electrocardiograph warrants an echocardiography evaluation for a patient with recurrent syncope.

- In young patients with recurrent syncope, we should consider structural heart disease or congenital heart disease as one of our differential diagnoses. 


\section{References}

1. Cardoso FB, Cardoso MB, Nishimura RI, et al. Atrial septal defect and pulmonary hypertension in professional soccer player. Arq Bras Cardiol. 2010 Aug;95(2):e38-9.

2. Diaconu C. Atrial septal defect in an elderly woman-A case report. J Med Life. 2011;4(1):91-93.

3. Mikhalkova D, Fenstad ER, Miller WL. 34-year-old man with exertional syncope, dyspnea, and chest pain. Mayo Clin Proc. 2013;88(7):756-760. doi:10.1016/j. mayocp.2012.09.012

4. Supomo S, Hartopo AB, Anggrahini DW, et al. Large atrial septal defect closure in a patient with severe pulmonary arterial hypertension. Korean J Thorac Cardiovasc Surg. 2017;50(5):378-381.
5. Post MC. Association between pulmonary hypertension and an atrial septal defect. Neth Heart J. 2013;21(7-8):331-332.

6. Wu RH, Li DF, Tang WT, et al. Atrial septal defect in a patient with congenital disorder of glycosylation type 1a: A case report. J Med Case Rep. 2018;12(1):17. doi:10.1186/ s13256-017-1528-4

7. Krishnamoorthy S, Butt M, Lip GY. Asymptomatic hypoxia in a young pregnant lady--Unusual presentation of atrial septal defect. Int J Cardiol. 2010 Aug 20;143(2):e346. doi: 10.1016/j.ijcard.2008.12.039. Epub 2009 Jan 21. PMID: 19157590.

8. Disenhoused RB, Anderson RC, Adams P Jr, et al. Atrial septal defect in infants and children. J Pediatr. 1954 Mar;44(3):269-89. doi: 10.1016/s0022-3476(54)80316-7. PMID: 13143453.
9. Forlemu AN, Ajmal M, Saririan M. Atrial septal defect with Eisenmenger syndrome: A rare presentation. Case Rep Cardiol. 2020;2020:8681761. Published 2020 Mar 9 doi:10.1155/2020/8681761

10. Basha A, Krishnan S, Gujral J, et al. Pulmonary arterial hypertension with frequent syncope: Clinical case presentation. J Pract Cardiovasc Sci 2018;4:126-31 